\title{
EIGENVALUE APPROACH TO NANOBEAM IN MODIFIED COUPLE STRESS THERMOELASTIC WITH THREE-PHASE-LAG MODEL INDUCED BY RAMP TYPE HEATING
}

\author{
RAJNEESH KUMAR \\ Kurukshetra University, Department of Mathematics, Kurukshetra, India \\ e-mail: rajneesh_kuk@rediffmail.com \\ SHALOO DEVI \\ Himachal Pradesh University, Department of Mathematics and Statistics, Shimla, India \\ e-mail: shaloosharma2673@gmail.com
}

\begin{abstract}
This article deals with the study of a thermoelastic nanobeam in a modified couple stress theory subjected to ramp-type heating. The mathematical model is prepared for the nanobeam in thermoelastic three-phase-lag. The Laplace transform and the eigenvalue approach are used to find the displacement component, lateral deflection, temperature change and axial stress of the thermoelastic beam. The general algorithm of the inverse Laplace transform is developed to compute results numerically. The comparison of three-phase-lag, dual-phaselag and GN-III (1993) models are represented, and their illustration is depicted graphically. This study finds the applications in engineering, medical science, sensors, etc.
\end{abstract}

Keywords: modified couple stress thermoelastic, eigenvalue approach, nanobeam

\section{Introduction}

Cosserat and Cosserat (1909) developed a mathematical model for a couple stress theory in which kinematical quantities are the displacement and material microrotation. Yang et al. (2002) proposed a modified couple stress theory in which the couple stress tensor was symmetric and required only one material length parameter to capture the size effect which was caused by micro-structure. Various authors studied different problems in a modified couple stress theory (2008, 2011-2015).

Tzou (1995a,b, 1997) proposed a dual-phase-lag model by modifying the classical fourier law by an approximation with two different time translations: a phase-lag of the heat flux $\tau_{q}$ and a phase-lag of the temperature gradient $\tau_{\theta}$. Tzou (1995b) supported that model by experimental results. A review of five theories of thermoelasticity was given by Hetnarski and Ignaczak (1999). Roychoudhuri (2007) developed a three-phase-lag model for a thermoelastic material. In that model, the Fourier law of heat conduction was modified by introducing three different phase-lags for the heat flux vector, temperature gradient and thermal displacement component gradient. Quintanilla and Racke (2008) investigated stability of the three-phase-lag heat conduction equation and the relations among three material parameters. Kumar et al. (2012) studied wave propagation in an anisotropic viscoelastic medium with the three-phase-lag model of thermoelasticity. Sur and Kanoria (2014) examined vibration of a gold nanobeam induced by a ramp-type laser pulse under the three-phase-lag model.

The significance of using the eigenvalue approach is to reduce the problem on the vector-matrix differential equation to algebraic eigenvalue problems. Thus the solutions for the field variables are obtained by determining eigenvalues and the corresponding eigenvectors. In this approach, the physical quantities are directly involved in the formulation of the problem and, 
as such, the boundary and initial conditions can be applied directly. The problem of micropolar thermoelasticity without energy dissipation by employing the eigenvalue approach was studied by Kumar et al. (2007). Zang and Fu (2012) constructed a new beam model for a viscoelastic micro-beam based on a modified couple stress theory.

Abouelregal and Zenkour (2014) investigated the problem of an axially moving microbeam subjected to a sinusoidal pulse heating and an external transverse excitation with one relaxation time using the Laplace transform. Zenkour and Abouelregal (2015) investigated the problem of a thermoviscoelastic orthotropic continuum with a cylindrical hole and variable thermal conductivity under three-phase-lag model and solved the physical quantities by the Laplace transform technique. The effects of hall current and rotation in a modified couple stress theory subjected to the ramp type loading in the context of theory of generalized thermoelastic diffusion was presented by Kumar and Devi (2015). Thermoelastic interaction in a thermally conducting cubic crystal subjected to the ramp-type heating was investigated by Abbas et al. (2015). Reddy et al. (2016) discussed the problem of functionally graded circular plates with the modified couple stress theory by using the finite element method. On the basis of global local theory, a model for the composite laminated Reddy plate of a new modified couple-stress theory was developed by Chen and Wang (2016). Zenkour and Abouelregal (2016) discussed vibration of functionally graded microbeams by using the Green-Naghdi thermoelasticity theory (1993) and the Laplace transform.

The present investigation deals with a thermoelastic nanobeam in the modified couple stress theory induced by the ramp-type heating in the three-phase-lag model. The non-dimensional equations are written in form of the Laplace transform which is solved by the eigenvalue approach. The expressions of the displacement component, lateral deflection, temperature change and axial stress are computed numerically and then represented graphically. Particular cases of interest are deduced from the present investigation.

\section{Basic equations}

Following Yang et al. (2002) and Roychoudhuri (2007), the constitutive relations, equations of motion and the equation of heat conduction in the modified couple stress generalized thermoelasticity with three-phase-lag model in the absence of body forces are:

- constitutive relations

$$
\begin{aligned}
t_{i j} & =\lambda e_{k k} \delta_{i j}+2 \mu e_{i j}-\frac{1}{2} e_{k i j} m_{l k, l}-\beta_{1} T \delta_{i j} \\
\chi_{i j} & =\frac{1}{2}\left(\omega_{i, j}+\omega_{j, i}\right) \quad \omega_{i}=\frac{1}{2} e_{i p q} u_{q, p}
\end{aligned}
$$

- equations of motion

$$
\left(\lambda+\mu+\frac{\alpha}{4} \Delta\right) \nabla(\nabla \cdot \mathbf{u})+\left(\mu-\frac{\alpha}{4} \Delta\right) \nabla^{2} \mathbf{u}-\beta_{1} \nabla T=\rho \ddot{\mathbf{u}}
$$

- equation of heat conduction with three-phase-lag

$$
\left[K^{*}\left(1+\tau_{\nu} \frac{\partial}{\partial t}\right)+K \frac{\partial}{\partial t}\left(1+\tau_{T} \frac{\partial}{\partial t}\right)\right] \Delta T=\left(1+\tau_{q} \frac{\partial}{\partial t}+\frac{\tau_{q}^{2}}{2} \frac{\partial^{2}}{\partial t^{2}}\right)\left(\rho c_{e} \frac{\partial^{2} T}{\partial t^{2}}+T_{0} \beta_{1} \frac{\partial^{2}}{\partial t^{2}}(\nabla \cdot \mathbf{u})\right)
$$

where $t_{i j}$ are components of the stress tensor, $\lambda$ and $\mu$ are Lame's constants, $\delta_{i j}$ is Kronecker's delta, $e_{i j}=\left(u_{i, j}+u_{j, i}\right) / 2$ are components of the strain tensor, $e_{i j k}$ is the alternate tensor, $m_{i j}$ are components of the couple-stress, $\beta_{1}=(3 \lambda+2 \mu) \alpha_{t}$. Here $\alpha_{t}$ are coefficients of linear thermal expansion and diffusion, respectively, $T$ is temperature change, $\chi_{i j}$ is symmetric curvature, $\omega_{i j}=\left(u_{j, i}-u_{i, j}\right) / 2$ are components of rotation, $\boldsymbol{\omega}_{i}$ is the rotational vector, $\alpha$ is the couple 
stress parameter and $\mathbf{u}=\left[u_{1}, u_{2}, u_{3}\right]$ is the displacement component, $\rho$ is density, $\Delta$ is the Laplacian operator, $\nabla$ is del operator. $K$ is the coefficient of thermal conductivity, $K^{*}$ is the material characteristic constant of the theory, $c_{e}$ is the specific heat at a constant strain, $T_{0}$ is the reference temperature assumed to be such that $T / T_{0} \ll 1 . \tau_{T}, \tau_{q}$ and $\tau_{\nu}$ are the phase lags of the temperature gradient, of the heat flux and of the thermal displacement component gradient, respectively, such that $\tau_{\nu}<\tau_{T}<\tau_{q}$.

\section{Formulation of the problem}

Consider a homogeneous, isotropic, rectangular modified couple stress thermoelastic beam having dimensions of length $(0 \leqslant x \leqslant L)$, width $(-d / 2 \leqslant y \leqslant d / 2)$ and thickness $(-h / 2 \leqslant z \leqslant h / 2)$ (Fig. 1). Let us take the $x$-axis along length of the beam, the $y$-axis along width and $z$-axis along thickness, representing the axis of material symmetry. Therefore, any plane cross-section initially perpendicular to the axis of the beam remains plane and perpendicular to the neutral surface during bending. According to the Euler-Bernoulli theory for a small deflection in a simple bending problem, the displacement components are given by

$$
u(x, y, z, t)=-z \frac{\partial w}{\partial x} \quad v(x, y, z, t)=0 \quad w(x, y, z, t)=w(x, t)
$$

where $w(x, t)$ is lateral deflection of the beam and $t$ is time. The one-dimension stress component $t_{x}$, with the aid of equations $(2.1)_{1}$ and (3.1), yields

$$
(3.2) t_{x}=-(\lambda+2 \mu) z \frac{\partial^{2} w}{\partial x^{2}}-\beta_{1} T
$$

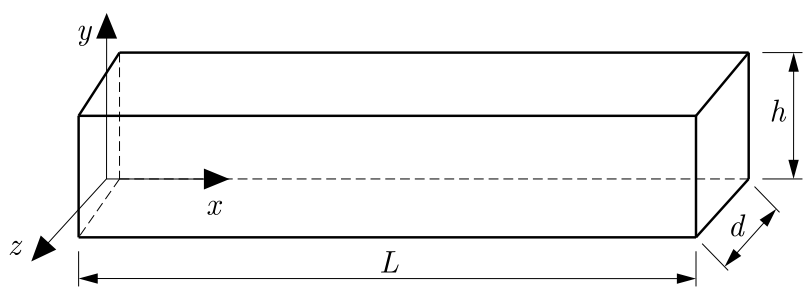

Fig. 1. Schematic figure of the beam

The flexural moment of the cross-section of the beam is given by

$$
M=M_{\sigma}+M_{m}=d\left(\int_{-h / 2}^{h / 2} t_{x} z d z+\int_{-h / 2}^{h / 2} m_{x y} d z\right)
$$

where $M_{\sigma}$ and $M_{m}$ are components of the bending moment due to the classic stress and couple stress tensors, respectively.

Making use of the value of $t_{x}$ and $m_{x y}$ from (3.2) and (2.1) $)_{2}$ in (3.3), with the aid of (3.1), yield

$$
M=-\left[(\lambda+2 \mu) \frac{d h^{3}}{12}+\alpha A\right] \frac{\partial^{2} w}{\partial x^{2}}-\beta_{1} d \int_{-h / 2}^{h / 2} T z d z
$$

Following Rao (2007), the equation of transverse motion of the beam is given by

$$
\frac{\partial^{2} M}{\partial x^{2}}-\rho A \frac{\partial^{2} w}{\partial t^{2}}=0
$$

where $A=d h$ is the cross-sectional area of the beam. 
For a very thin beam, assuming that the temperature increment varies in terms of the $\sin (p z)$ function along thickness of the beam, where $p=\pi / h$ as

$$
T(x, z, t)=T_{1}(x, t) \sin (p z)
$$

Substituting the value of $M$ from (3.4) into equation (3.5), with the aid of equation (3.6), yields

$$
\left[(\lambda+2 \mu) \frac{d h^{3}}{12}+\alpha A\right] \frac{\partial^{4} w}{\partial x^{4}}+\beta_{1} d \frac{\partial^{2} T_{1}}{\partial x^{2}} \int_{-h / 2}^{h / 2} z \sin (p z) d z+\rho A \frac{\partial^{2} w}{\partial t^{2}}=0
$$

Multiplying heat conduction equation (2.3), after using equation (3.1), by $z$ and integrating them with respect to the interval $(-h / 2, h / 2)$, and with the use of equation (3.6), we obtain

$$
\begin{aligned}
& \left.\left[K^{*}\left(1+\tau_{\nu} \frac{\partial}{\partial t}\right)+K \frac{\partial}{\partial t}\left(1+\tau_{T}\right) \frac{\partial}{\partial t}\right)\right]\left(\frac{\partial^{2} T_{1}}{\partial x^{2}}-p^{2} T_{1}\right) \\
& \quad=\left(1+\tau_{q} \frac{\partial}{\partial t}+\frac{\tau_{q}^{2}}{2} \frac{\partial^{2}}{\partial t^{2}}\right)\left(\rho c_{e} \frac{\partial^{2} T_{1}}{\partial t^{2}}-\frac{\beta_{1} T_{0} p^{2} h^{3}}{24} \frac{\partial^{4} w}{\partial x^{2} \partial t^{2}}\right)
\end{aligned}
$$

To facilitate solution, the following dimensionless quantities are introduced

$$
\begin{array}{lll}
x^{\prime}=\frac{x}{L} & \left(z^{\prime}, u^{\prime}, w^{\prime}\right)=\frac{(z, u, w)}{h} & \left(\tau_{\nu}^{\prime}, \tau_{T}^{\prime}, \tau_{q}^{\prime}, t^{\prime}\right)=\frac{\left(\tau_{\nu}, \tau_{T}, \tau_{q}, t\right) \nu}{L} \\
T_{1}^{\prime}=\frac{\beta_{1} T_{1}}{E} & \left(M^{\prime}, M_{T}^{\prime}\right)=\frac{\left(M, M_{T}\right)}{d E h^{2}} & t_{x}^{\prime}=\frac{t_{x}}{E} \\
\nu^{2}=\frac{E}{\rho} & K^{*}=\frac{c_{e}(\lambda+2 \mu)}{4} &
\end{array}
$$

Making use of equation (3.9) in (3.7) and (3.8), after surpassing the primes, we obtain

$$
\begin{aligned}
& \frac{\partial^{4} w}{\partial x^{4}}+a_{1} \frac{\partial^{2} T_{1}}{\partial x^{2}}+a_{2} \frac{\partial^{2} w}{\partial t^{2}}=0 \\
& {\left[a_{3}\left(1+\tau_{\nu} \frac{\partial}{\partial t}\right)+\frac{\partial}{\partial t}\left(1+\tau_{T} \frac{\partial}{\partial t}\right)\right]\left(\frac{\partial^{2} T_{1}}{\partial x^{2}}-a_{4} T_{1}\right)} \\
& \quad=\left(1+\tau_{q} \frac{\partial}{\partial t}+\frac{\tau_{q}^{2}}{2} \frac{\partial^{2}}{\partial t^{2}}\right)\left(a_{5} \frac{\partial^{2} T_{1}}{\partial t^{2}}-a_{6} \frac{\partial^{2} w}{\partial x^{2} \partial t^{2}}\right)=0
\end{aligned}
$$

where

$$
\begin{array}{lll}
a_{1}=\frac{2 d E L}{p^{2}\left[(\lambda+2 \mu) \frac{d h^{3}}{12}+\alpha A\right]} & a_{2}=\frac{\rho A \nu^{2} L^{2}}{(\lambda+2 \mu) \frac{d h^{3}}{12}+\alpha A} & a_{3}=\frac{K^{*} L}{K \nu} \\
a_{4}=\frac{p^{2}}{L^{2}} & a_{5}=\frac{\rho c_{e} \nu L}{K} & a_{6}=\frac{\beta_{1}^{2} T_{0} \nu p^{2} h^{3}}{24 K E}
\end{array}
$$

\section{Problem solution}

The Laplace transform is defined as

$$
L\{f(t)\}=\int_{0}^{\infty} e^{-s t} f(t) d t=\bar{f}(s)
$$

where $s$ is the Laplace transform parameter. 
Applying the Laplace transform defined by equation (4.1) to equations (3.10) and (3.11), gives

$$
\frac{d^{4} \bar{w}}{d x^{4}}+a_{1} \frac{d^{2} \bar{T}_{1}}{d x^{2}}+a_{2} s^{2} \bar{w}=0 \quad \frac{d^{2} \bar{T}_{1}}{d x^{2}}-a_{4} \bar{T}_{1}=a_{7} s^{2} \bar{T}_{1}-a_{8} s^{2} \frac{d^{2} \bar{w}}{d x^{2}}
$$

where

$$
\begin{array}{rlrl}
\bar{\tau}_{q} & =1+\tau_{q} s+\frac{\tau_{q}^{2}}{2} s^{2} & \bar{\tau}_{\nu}=1+\tau_{\nu} s & \bar{\tau}_{T}=1+\tau_{T} s \\
a_{7}=\frac{a_{5} \bar{\tau}_{q}}{a_{3} \bar{\tau}_{\nu}+s \bar{\tau}_{T}} & a_{8}=\frac{a_{6} \bar{\tau}_{q}}{a_{3} \bar{\tau}_{\nu}+s \bar{\tau}_{T}} &
\end{array}
$$

The set of equations (4.2) can be written as

$$
\frac{d^{2} \bar{v}}{d x^{2}}=-a_{9} \bar{w}+a_{10} \bar{v}-a_{11} \bar{T}_{1} \quad \frac{d^{2} \bar{T}_{1}}{d x^{2}}=-a_{12} \bar{v}+a_{13} \bar{T}_{1}
$$

where

$$
\begin{array}{lll}
\frac{d^{2} \bar{w}}{d x^{2}}=\bar{v} & a_{9}=a_{2} s^{2} & a_{10}=a_{1} a_{8} s^{2} \\
a_{11}=a_{1}\left(a_{4}+a_{7} s^{2}\right) & a_{12}=a_{8} s^{2} & a_{13}=a_{4}+a_{7} s^{2}
\end{array}
$$

The system of equations (4.4) can be written in a matrix form as

$$
D \mathbf{V}(x, s)=\mathbf{A V}(x, s)
$$

where

$$
\mathbf{V}=\left[\begin{array}{c}
\mathbf{U} \\
D \mathbf{U}
\end{array}\right] \quad \mathbf{U}=\left[\begin{array}{c}
\bar{w} \\
\bar{v} \\
\bar{T}_{1}
\end{array}\right] \quad \mathbf{A}=\left[\begin{array}{cc}
\mathbf{O} & \mathbf{I} \\
\mathbf{A}_{1} & \mathbf{O}
\end{array}\right] \quad \mathbf{A}_{1}=\left[\begin{array}{ccc}
0 & 1 & 0 \\
-a_{9} & a_{10} & -a_{11} \\
0 & -a_{12} & a_{13}
\end{array}\right]
$$

and $D=d / d z, \mathbf{I}$ is the identity matrix of the order $3, \mathbf{O}$ is a null matrix of the order 3 .

We take the solution to equation (4.4) as

$$
\mathbf{V}(x, s)=\mathbf{X}_{r}(x, s) \mathrm{e}^{\lambda z}
$$

such that

$$
\mathbf{A}(x, s) \mathbf{V}(x, s)=\lambda \mathbf{V}(x, s)
$$

which leads to the eigenvalue approach. The characteristic equation of the matrix $\mathbf{A}$ can be written as

$$
\lambda^{6}-G_{1} \lambda^{4}+G_{2} \lambda^{2}-G_{3}=0
$$

where

$$
G_{1}=a_{10}+a_{13} \quad G_{2}=a_{9}+a_{10} a_{13}-a_{11} a_{12} \quad G_{3}=a_{9} a_{13}
$$

The characteristic roots of equation (4.6) are also the eigenvalues of the matrix $\mathbf{A}$. The eigenvectors $\mathbf{X}(x, s)$ corresponding to the eigenvalue $\lambda_{r}$ can be determined by solving the homogeneous equations

$$
(\mathbf{A}-\lambda \mathbf{I}) \mathbf{X}(x, s)=\mathbf{0}
$$


The set of eigenvectors $\mathbf{X}_{r}(x, s)$ may be obtained as

$$
\mathbf{X}_{r}(x, s)=\left[\begin{array}{l}
\mathbf{X}_{r 1}(x, s) \\
\mathbf{X}_{r 2}(x, s)
\end{array}\right] \quad \mathbf{X}_{r 1}(x, s)=\left[\begin{array}{c}
b_{r} \\
c_{r} \\
d_{r}
\end{array}\right] \quad \mathbf{X}_{r 2}(x, s)=\lambda_{r} \mathbf{X}_{r 1}(x, s)
$$

for $\lambda=\lambda_{r}, r=1,2,3$ and

$$
\mathbf{X}_{j}(x, s)=\left[\begin{array}{c}
\mathbf{X}_{j 1}(x, s) \\
\mathbf{X}_{j 2}(x, s)
\end{array}\right] \quad \mathbf{X}_{j 1}(x, s)=\left[\begin{array}{c}
b_{r} \\
c_{r} \\
d_{r}
\end{array}\right] \quad \mathbf{X}_{j 2}(x, s)=\lambda_{j} \mathbf{X}_{j 1}(x, s)
$$

for $j=r+4, \lambda=-\lambda_{r}, r=1,2,3$ and

$$
b_{r}=-a_{11} \quad c_{r}=-a_{11} \lambda_{r}^{2} \quad d_{r}=\lambda_{r}^{4}-a_{10} \lambda_{r}^{2}+a_{9}
$$

The solution to equation (4.6) reduces to

$$
\mathbf{V}=\sum_{r=1}^{3} B_{r} \mathbf{X}_{r}(x, s) \mathrm{e}^{-\lambda_{r} x}+\sum_{r=1}^{3} B_{r+3} \mathbf{X}_{r+3}(x, s) \mathrm{e}^{\lambda_{r} x}
$$

where $B_{i}(i=1, \ldots, 6)$ are arbitrary constants.

Thus, the field quantities can be written as

$$
\left(\bar{w}, \bar{v}, \bar{T}_{1}\right)(x, s)=\sum_{r=1}^{3}\left(b_{r}, c_{r}, d_{r}\right) B_{r} \mathrm{e}^{-\lambda_{r} x}+\sum_{j=1}^{3}\left(b_{j+3}, c_{j+3}, d_{j+3}\right) B_{j+3} \mathrm{e}^{\lambda_{j} x}
$$

\section{Initial and boundary conditions}

Both initial and boundary conditions should be considered to solve the problem. The initial conditions of the problem are taken in the form as

$$
\left.w(x, t)\right|_{t=0}=\left.\frac{\partial w(x, t)}{\partial t}\right|_{t=0}=\left.0 \quad T_{1}(x, t)\right|_{t=0}=\left.\frac{\partial T_{1}(x, t)}{\partial t}\right|_{t=0}=0
$$

Let us consider a nanobeam with both ends are simply supported

$$
w(0, t)=0 \quad \frac{\partial^{2} w(0, t)}{\partial x^{2}}=0 \quad w(L, t)=0 \quad \frac{\partial^{2} w(L, t)}{\partial x^{2}}=0
$$

We consider the side of the nanobeam $x=0$ being thermally loaded by ramp-type heating incidents into the surface of the nanobeam

$$
T_{1}(0, t)=g_{0} \begin{cases}0 & t \leqslant 0 \\ t / t_{0} & 0<t \leqslant t_{0} \\ 1 & t>t_{0}\end{cases}
$$

where $t_{0}$ is a non-negative constant called the ramp type parameter and $g_{0}$ is a constant.

We also assume that the other side of the nanobeam $x=L$ is thermally insulated, and there is no variation of temperature on it, which this means that the following relation will be satisfied

$$
\frac{d T_{1}(L, t)}{d x}=0
$$


Applying the Laplace transform defined by equation (4.1) to boundary conditions (5.2)-(5.4), we obtain

$$
\begin{array}{llll}
\bar{w}(0, s)=0 & \frac{d^{2} \bar{w}(0, s)}{d x^{2}}=0 & \bar{T}_{1}(0, s)=g_{0}\left(\frac{1-\mathrm{e}^{-s t_{0}}}{t_{0} s^{2}}\right) \\
\bar{w}(1, s)=0 & \frac{d^{2} \bar{w}(1, s)}{d x^{2}}=0 & \frac{d \bar{T}_{1}(1, s)}{d x}=0
\end{array}
$$

The values of displacement $\bar{u}$ and axial stress $\bar{t}_{x}$ are then obtained

$$
\begin{gathered}
\bar{u}(x, s)=z\left(\sum_{i=1}^{3} \lambda_{i} b_{i} B_{i} \mathrm{e}^{-\lambda_{i} x}-\sum_{i=1}^{3} \lambda_{i} b_{i+3} B_{i+3} \mathrm{e}^{\lambda_{i} x}\right) \\
\bar{t}_{x}(x, s)=-\left[\sum_{i=1}^{3}\left(\frac{\lambda+2 \mu}{E} z \lambda_{i}^{2} b_{i}+d_{i} \sin (p z)\right) B_{i} \mathrm{e}^{-\lambda_{i} x}\right. \\
\left.+\sum_{i=1}^{3}\left(\frac{\lambda+2 \mu}{E} z \lambda_{i}^{2} b_{i+3}+d_{i+3} \sin (p z)\right) B_{i+3} \mathrm{e}^{\lambda_{i} x}\right]
\end{gathered}
$$

Making use of the value of $\bar{w}$ and $\bar{T}_{1}$ from (4.11) in boundary conditions (5.5), with the aid of equations (5.6), after some calculations, we find the expressions of the displacement component, lateral deflection, temperature change and axial stress of the beam as

$$
\begin{aligned}
& (\bar{u}, \bar{w})(x, s)=\sum_{i=1}^{3}\left(z \lambda_{i}, 1\right) b_{i} B_{i} \mathrm{e}^{-\lambda_{i} x}+\sum_{i=1}^{3}\left(-\lambda_{i}, 1\right) b_{i+3} B_{i+3} \mathrm{e}^{\lambda_{i} x} \\
& \left(\bar{T}_{1}, \bar{t}_{x}\right)(x, s)=\sum_{i=1}^{3}\left(d_{i}, M_{i}\right) B_{i} \mathrm{e}^{-\lambda_{i} x}+\sum_{i=1}^{3}\left(d_{i+3}, M_{i+3}\right) B_{i+3} \mathrm{e}^{\lambda_{i} x}
\end{aligned}
$$

where

$$
B_{i}=\frac{\boldsymbol{\Delta}_{i}}{\boldsymbol{\Delta}} \quad i=1, \ldots, 6
$$

and

$$
\begin{aligned}
& \sum_{i=1}^{3} M_{i}=-\left(\frac{\lambda+2 \mu}{E} z \lambda_{i}^{2} b_{i}+d_{i} \sin (p z)\right) \quad \sum_{i=1}^{3} M_{i+3}=-\left(\frac{\lambda+2 \mu}{E} z \lambda_{i}^{2} b_{i+3}+d_{i+3} \sin (p z)\right) \\
& \boldsymbol{\Delta}=\left[\begin{array}{cccccc}
b_{1} & b_{2} & b_{3} & b_{4} & b_{5} & b_{6} \\
b_{1} \mathrm{e}^{-\lambda_{1}} & b_{2} \mathrm{e}^{-\lambda_{2}} & b_{3} \mathrm{e}^{-\lambda_{3}} & b_{4} \mathrm{e}^{\lambda_{2}} & b_{5} \mathrm{e}^{\lambda_{2}} & b_{6} \mathrm{e}^{\lambda_{2}} \\
b_{1} \lambda_{1}^{2} & b_{2} \lambda_{2}^{2} & b_{3} \lambda_{3}^{2} & b_{4} \lambda_{1}^{2} & b_{5} \lambda_{2}^{2} & b_{6} \lambda_{3}^{2} \\
b_{1} \lambda_{1}^{2} \mathrm{e}^{-\lambda_{1}} & b_{2} \lambda_{2}^{2} \mathrm{e}^{-\lambda_{2}} & b_{3} \lambda_{3}^{2} \mathrm{e}^{-\lambda_{3}} & b_{4} \lambda_{1}^{2} \mathrm{e}^{-\lambda_{1}} & b_{5} \lambda_{2}^{2} \mathrm{e}^{-\lambda_{2}} & b_{6} \lambda_{3}^{2} \mathrm{e}^{-\lambda_{3}} \\
d_{1} & d_{2} & d_{3} & d_{4} & d_{5} & d_{6} \\
-d_{1} \lambda_{1} \mathrm{e}^{-\lambda_{1}} & -d_{2} \lambda_{2} \mathrm{e}^{-\lambda_{2}} & -d_{3} \lambda_{3} \mathrm{e}^{-\lambda_{3}} & -d_{4} \lambda_{1} \mathrm{e}^{\lambda_{1}} & d_{5} \lambda_{2} \mathrm{e}^{\lambda_{2}} & d_{6} \lambda_{3} \mathrm{e}^{\lambda_{3}}
\end{array}\right]
\end{aligned}
$$

where $\boldsymbol{\Delta}_{i}(i=1, \ldots, 6)$ are obtained by replacing the $i$-th column with $\left[0,0,0,0, g_{0}((1-\right.$ $\left.\left.\left.\mathrm{e}^{-s t_{0}}\right) /\left(t_{0} s^{2}\right)\right), 0\right]^{\mathrm{T}}$ in $\boldsymbol{\Delta}_{i}$.

\section{Particular cases}

(i) Dual-phase-lag model

If $K^{*}=\tau_{\nu}=0$, in equations (5.7), we obtain the corresponding results for modified couple stress thermoelastic materials with the dual-phase-lag model of thermoelasticity. 
(ii) GN-III model

In the absence of $\tau_{\nu}=\tau_{T}=\tau_{q}=\tau_{q}^{2}=0$ in equations (5.7), we obtain the corresponding results for modified couple stress thermoelastic materials with energy dissipation in the context of GN-III theory of thermoelasticity.

(iii) If we take $\alpha=0$ in equations (5.7), we obtain the corresponding results for thermoelastic materials with the three-phase-lag model of thermoelasticity. Our results in a special case are similar to those obtained by Sur and Kanoria (2014).

\section{Inversion of the Laplace transform}

We have obtained solutions for the displacement component, lateral deflection, temperature change and axial stress in the Laplace transform domain $(x, s)$. We shall now briefly outline the numerical inversion method used to find the solution in the physical domain. Let $\bar{f}(s)$ be the Laplace transform of a function $f(t)$. To obtain the solution of the problem in the physical domain, we invert the Laplace transform by using the method described by Kumar (2016).

\section{Numerical results and discussion}

We have chosen gold $(\mathrm{Au})$ as the material for numerical computations. The physical data for gold are given by Sur and Kanoria (2014): $\lambda=198 \mathrm{GPa}, \mu=27 \mathrm{GPa}, \alpha_{t}=14.2 \cdot 10^{-6} \mathrm{~K}^{-1}$, $\rho=1930 \mathrm{~kg} / \mathrm{m}^{3}, T_{0}=0.293 \cdot 10^{3} \mathrm{~K}, \nu=0.44, K=200 \mathrm{~W} /(\mathrm{mK}), c_{e}=130 \mathrm{~J} /(\mathrm{kg} \mathrm{K})$, $\alpha=2.5 \mathrm{~kg} \mathrm{~m} / \mathrm{s}^{2}, t=1.5 \mathrm{~s}, \tau_{\nu}=0.02 \mathrm{~s}, \tau_{T}=0.03 \mathrm{~s}, \tau_{q}=0.04 \mathrm{~s}, g_{0}=1, t_{0}=0.2, L=1$, $d=1, h=10$.

Numerical computations have been carried out with the help of MATLAB software. By using this software, the displacement component, lateral deflection, temperature change, thermal stress, bending moment and axial stress with respect to distance are computed numerically and shown graphically in Figs. 2-7. In Figs. 2-4, the small dash line (- - ) corresponds to the three-phase-lag model (TPL), small dash line with the centre symbol (- - * - ) corresponds to the dual-phase-lag model (DPL) and a small dash line with the centre symbol (- - o - -) corresponds to the GN-III model respectively. Similarly, Figs. 5-7, the small dash line (- - -) corresponds to $t_{0}=0.2$, small dash line with the centre symbol $(--*--)$ corresponds to $t_{0}=0.4$, the small dash line with the centre symbol (- - o - -) corresponds to $t_{0}=0.6$.

Figure $2 \mathrm{a}$ shows the variation of the displacement component with respect to length of the beam for different models. The behavior and variation are similar for all the cases but with differences in their magnitudes. However, the values of the GN-III model are greater than compared to DPL and TPL models. Figure $2 \mathrm{~b}$ depicts the variation of lateral deflection with respect to length of the beam for the three-phase-lag, dual-phase-lag and GN-III models. It is observed that the lateral deflection decreases for smaller values of length and oscillates for higher values of length for all TPL, DPL and GN-III models.

Figure 3a presents the variation of axial stress with respect to length of the beam for TPL, DPL, GN-III models. As seen in the figure, the axial stress decreases smoothly in the whole region for all cases. Also, it is noticed that the axial stress has a large value for the three-phase-lag (TPL) and dual-phase-lag (DPL) thermoelastic beams as compared to that for the GN-III thermoelastic beam. Figure 3b shows the variation of temperature change with respect to length of the beam for different thermoelastic (TPL, DPL, GN-III) models. It is observed from the figure that the behavior and variation are oscillatory in nature with fluctuating values in all the cases. 
(a)

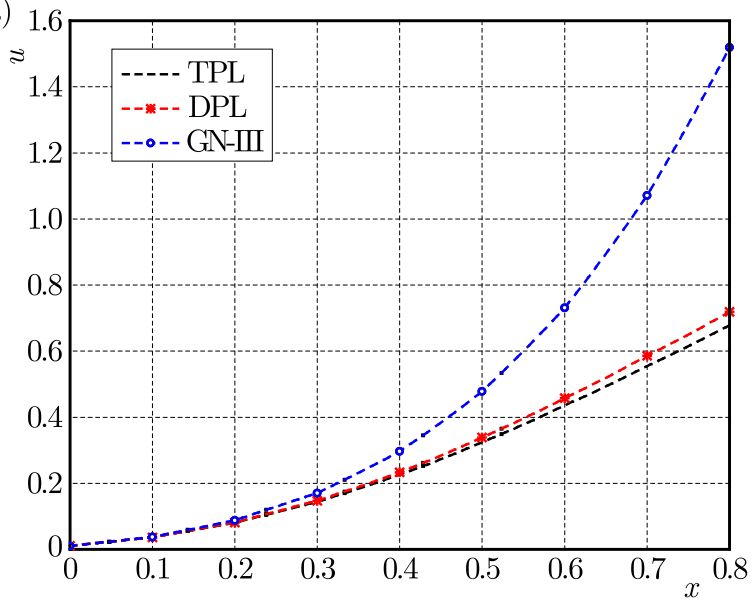

(b)

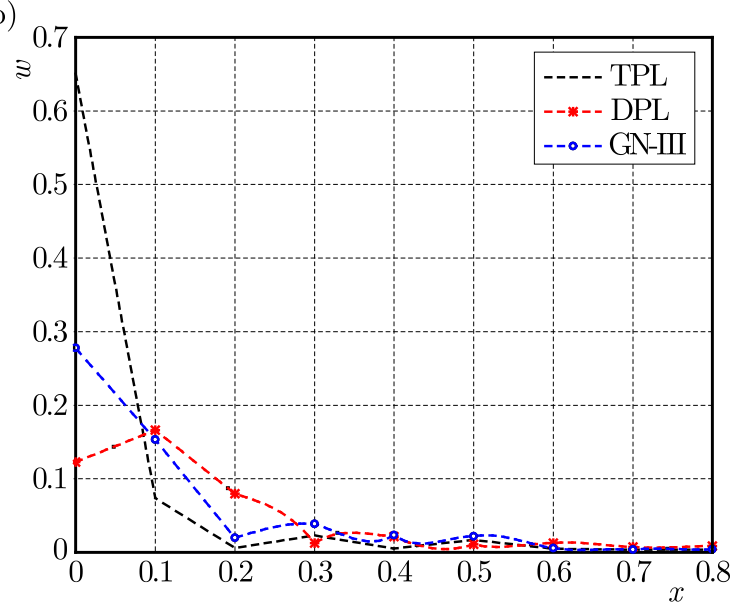

Fig. 2. (a) Displacement component $u$ and (b) lateral deflection $w$ with respect to distance $x$ for different phase lag theories of thermoelasticity

(a)

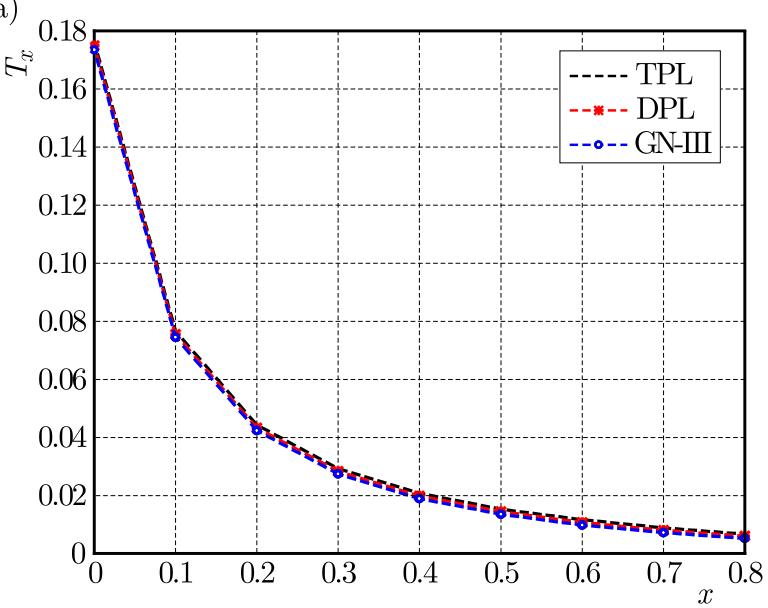

(b)

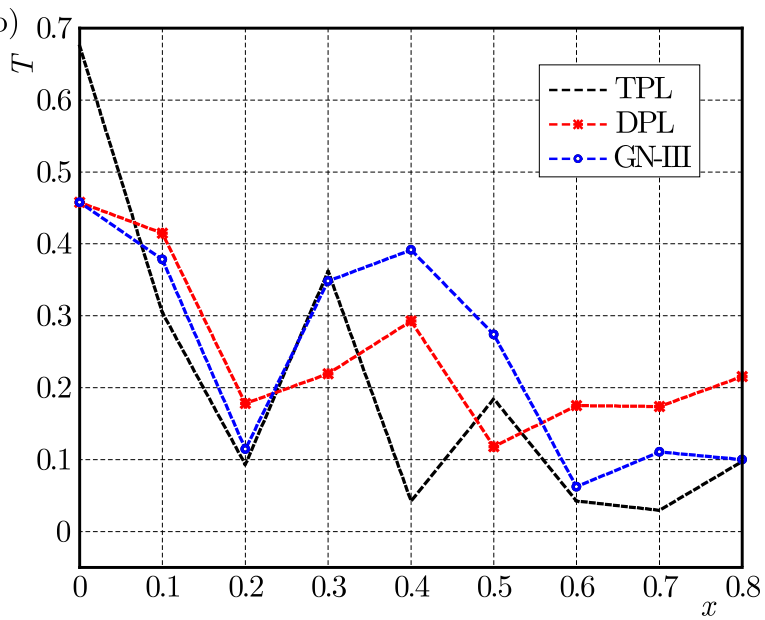

Fig. 3. (a) Axial stress $T_{x}$ and (b) temperature change $T$ with respect to distance $x$ for different phase lag theories of thermoelasticity

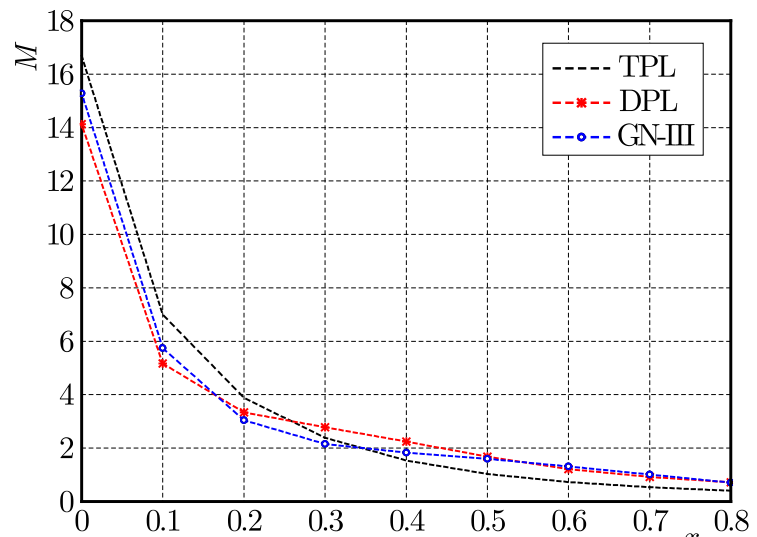

Fig. 4. Bending moment $M$ with respect to distance $x$ for different phase $e^{x}$ lag theories of thermoelasticity

Figure 4 shows the variation of bending moment with respect to length of the beam for different thermoelastic models. It is clearly seen in the figure that the value of bending moment decreases with a decrease in the value of length for all the cases of phase lag theories of thermoelasticity. Also, the value of bending moment is higher in the range $0 \leqslant x \leqslant 0.25$ for $t_{0}=0.2$ and smaller for $t_{0}=0.4,0.6$ in the remaining range. 
Figure 5a shows the variation of lateral deflection with respect to length of the beam for different values of the ramp type parameter. Initially, the lateral deflection decreases with a difference in the ramp type parameter up to $x \leqslant 0.3$ and then remains stable in the range $0.3<x \leqslant 0.8$. Figure $5 \mathrm{~b}$ presents the variation of temperature change with respect to length of the beam for different values of the ramp type parameter. The behavior and variation are oscillatory in nature for all the cases of the ramp type parameter.

(a)

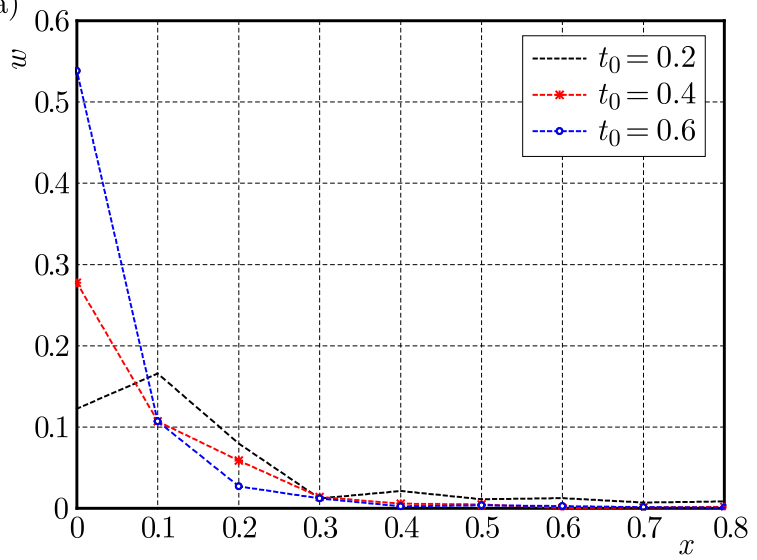

(b)

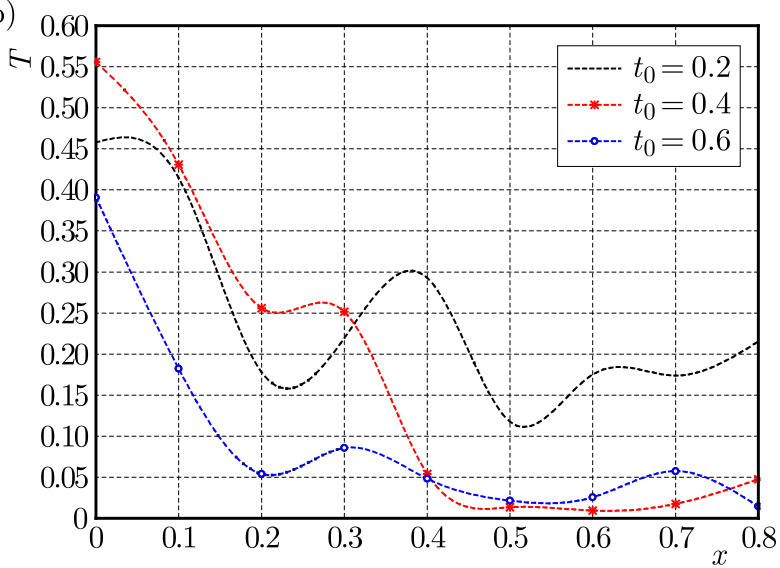

Fig. 5. (a) Lateral deflection $w$ and (b) temperature change $T$ with respect to length $x$ for different values of the ramp type parameter

Figure 6a shows the variation of axial stress with respect to length of the beam for different values of the ramp type parameter. The value of axial stress decreases monotonically with an increase in length. Also, the value of axial stress for $t_{0}=0.2$ is greater than that the ramp type parameter $t_{0}=0.4,0.6$.

Figure $6 \mathrm{~b}$ depicts the variation of displacement component with respect to length of the beam for different values of the ramp type parameter. The displacement component increases with an increase in length.

(a)

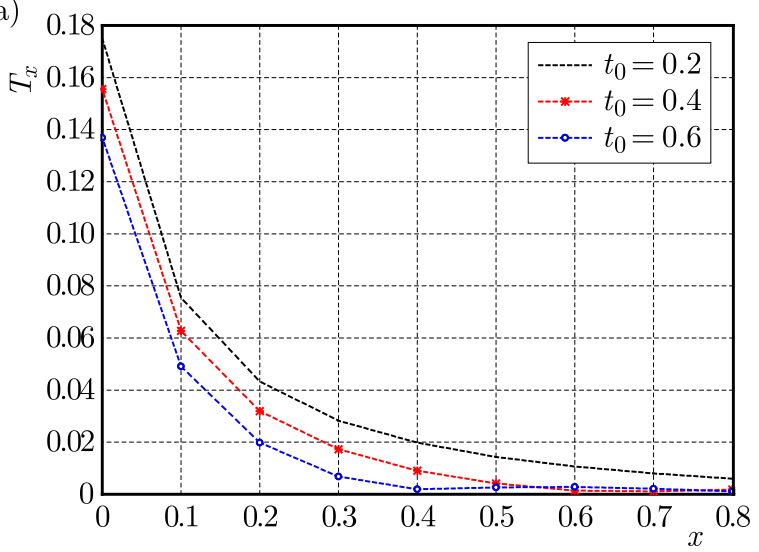

(b)

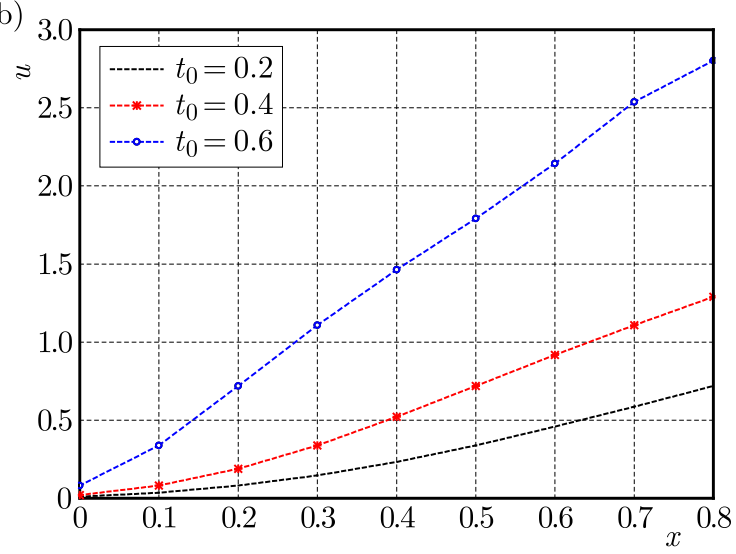

Fig. 6. (a) Axial stress $T_{x}$ and (b) displacement component $u$ with respect to distance $x$ for different values of the ramp type parameter

Figure 7a represents the variation of bending moment with respect to length of the beam for different values of the ramp type parameter. The bending moment smoothly decreases with a decrease in the value of length. The value of the bending moment is greater for $t_{0}=0.2$ up to the value of $x=0.25$ in comparison with that for $t_{0}=0.4,0.6$, but shows opposite behavior in the remaining range. Figure $7 \mathrm{~b}$ depicts the variation of thermal stress with respect to length of the beam for different values of the ramp type parameter. The oscillatory behavior is shown for 
all the cases of ramp type parameters. It is clear from the figure that the value of thermal stress for $t_{0}=0.6$ is less in the range $0 \leqslant x \leqslant 0.5$ but reversed behavior is shown for $t_{0}=0.2,0.4$ in the considered region.

(a)

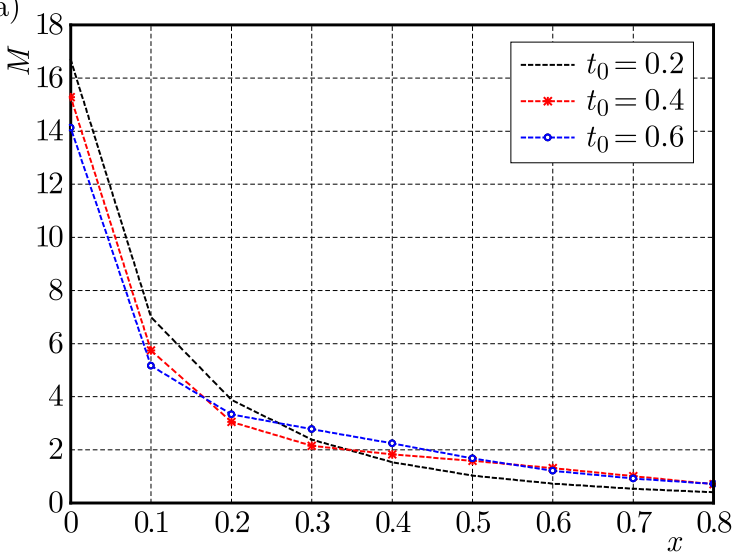

(b)

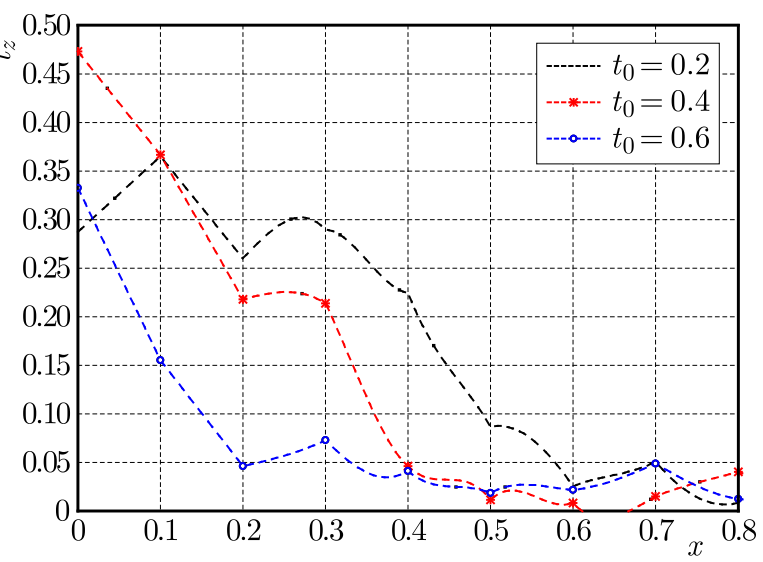

Fig. 7. (a) Bending moment $M$ and (b) thermal stress $t_{z}$ with respect to distance $x$ for different values of the ramp type parameter

\section{Conclusions}

In the present study, the effects of three-phase-lag, dual-phase-lag and GN-III on the displacement component, lateral deflection, axial stress, bending moment and temperature change are derived numerically and presented graphically. The effect of the ramp type parameter is shown graphically for lateral deflection, bending moment, displacement component, axial stress, thermal stress and temperature change. The Euler Bernoulli beam assumption and the Laplace transform technique are used to write the basic governing equations in form of vector-matrix differential equations which are then calculated by the eigenvalue approach. A numerical technique has been adopted to determine solutions in the physical domain. It is observed from the obtained figures that the displacement component increases with a increase in length for all the three-phase-lag, dual-phase-lag and GN-III models but opposite behavior is observed for the axial stress. It is also noticed that the values of displacement component and axial stress for the three-phase-lag thermoelastic model is greater in comparison with the dual-phase-lag and GN-III models. The lateral deflection and temperature change are oscillatory in nature but differ in their magnitude values for all the cases. The method used in the present study is applicable to a wide range of mathematical problems in the field of thermodynamics, thermoelasticity and couple stress theory. This study also find various applications to applied mathematics, mechanical engineering, geophysical and industrial sectors.

\section{References}

1. Abbas I.A., Kumar R., Rani L., 2015, Thermoelastic interaction in a thermally conducting cubic crystal subjected to ramp-type heating, Applied Mathematics and Computation, 254, 360-369

2. Abouelregal A.E., Zenkour A.M., 2014, Effect of phase lags on thermoelastic functionally graded microbeams subjected to ramp-type heating, Iranian Journal of Science and Technology: Transactions of Mechanical Engineering, 38, M2, 321-335

3. Asghari M., 2012, Geometrically nonlinear micro-plate formulation based on the modified couple stress theory, International Journal of Engineering Science, 51, 292-309 
4. Cosserat E., Cosserat F., 1909, Theory of Deformable Bodies, Hermann et Fils, Paris

5. Chen W., Li L., Xu M., 2011, A modified couple stress model for bending analysis of composite laminated beams with first order shear deformation, Composite Structures, 93, 2723-2732

6. Chen W., Wang Y., 2016, A model of composite laminated Reddy plate of the global-local theory based on new modified couple-stress theory, Mechanics of Advanced Materials and Structures, 23, $6,636-651$

7. Darijani H., Shahdadi A.H., 2015, A new shear deformation model with modified couple stress theory for microplates, Acta Mechanica, 226, 2773-2788

8. Green A.E., Naghdi P.M., 1993, Thermoelasticity without energy dissipation, Journal of Elasticity, 31, 189-209

9. Hetnarski R.B., Ignaczak J., 1999, Generalized themoelasticity, Journal of Thermal Stresses, 22, 451-476

10. Kumar R., 2016, Response of thermoelastic beam due to thermal source in modified couple stress theory, Computational Methods in Science and Technology, 22, 2, 87-93

11. Kumar R., Chawla V., ABBas I.A., 2012, Effect of viscosity on wave propagation in anisotropic thermoelastic medium with three-phase-lag model, Theoretical and Applied Mechanics, 39, 4, $313-341$

12. Kumar R., Devi S., 2015, Interaction due to Hall current and rotation in a modified couple stress elastic half-space due to ramp-type loading, Computational Methods in Science and Technology, 21, 4, 229-240, DOI:10.12921/cmst.2015.21.04.007.

13. Kumar R., Singh R., Chadha T.K., 2007, Eigenvalue approach to micropolar thermoelasticity without energy dissipation, Indian Journal of Mathematics, 49, 3, 355-369

14. Ma H.M., Gao X.L., Reddy J.N., 2008, A microstructure-dependent Timoshenko beam model based on a modified couple stress theory, Journal of the Mechanics and Physics of Solids, 56, 3379-3391

15. Mohammad-Abadi M., Daneshmehr A.R., 2014, Size dependent buckling analysis of micro beams based on modified couple stress theory with high order theories and general boundary conditions, International Journal of Engineering Science, 74, 1-14

16. Quintanilla R., Racke R.A., 2008, Note on stability in three-phase-lag heat conduction, International Journal of Heat Mass Transfer, 51, 1/2, 24-29

17. Rao S.S., 2007, Vibration of Continuous Systems, John Wiley \& Sons, Inc. Hoboken, New Jersey

18. Reddy J.N., Romanoff J., Loya J.A., 2016, Nonlinear finite element analysis of functionally graded circular plates with modified couple stress theory, European Journal of Mechanics A/Solids, 56, 92-104

19. Rezazadeh G., Vahdat A.S., Tayefeh-Rezaei S., Cetinkaya C., 2012, Thermoelastic damping in a micro-beam resonator using modified couple stress theory, Acta Mechanica, 223, 6, $1137-1152$

20. Roychoudhuri S.K., 2007, On a thermoelastic three-phase-lag model, Journal of Thermal Stresses, 30, 231-238

21. SimseK M., Reddy J.N., 2013, Bending and vibration of functionally graded microbeams using a new higher order beam theory and the modified couple stress theory, International Journal of Engineering Science, 64, 37-53

22. Sur A., Kanoria M., 2014, Vibration of a gold-nanobeam induced by ramp type laser pulse three-phase-lag model, International Journal of Applied Mathematics and Mechanics, 10, 5, $86-104$

23. Tzou D.Y., 1995a, A unified field approach for heat conduction from micro to macroscales, $A S M E$ Journal of Heat Transfer, 117, 8-16 
24. Tzou D.Y., 1995b, Experiments support for the lagging behaviour in heat propagation, Journal of Thermophysics and Heat Transfer, 9, 686-693

25. Tzou D.Y., 1997, Macro to Microscale Heat Transfer: the Lagging Behaviour, Series in Chemical ad Mechanical Engineering, Taylor \& Francis, Washington, DC

26. Yaghoub T.B., Fahimeh M., Hamed R., 2015, Free vibration analysis of size-dependent shear deformable functionally graded cylindrical shell on the basis of modified couple stress theory, Composite Structures, 120, 65-78

27. Yang F., Chong A.C.M., Lam D.C.C., Tong P., 2002, Couple stress based strain gradient theory for elasticity, International Journal of Solids and Structures, 39, 2731-2743

28. Yong-Gang W., Wen-Hui L., Liu N., 2015, Nonlinear bending and post-buckling of extensible microscale beams based on modified couple stress theory, Applied Mathematical Modelling, 39, $117-127$

29. ZANG J., FU Y., 2012, Pull-in analysis of electrically actuated viscoelastic microbeams based on a modified couple stress theory, Meccanica, 47, 1649-1658

30. Zenkour A.M., Abouelregal A.E., 2015, Effects of phase-lags in a thermoviscoelastic orthotropic continuum with a cylindrical hole and variable thermal conductivity, Archives of Mechanics, 67, $6,457-475$

31. Zenkour A.M., Abouelregal A.E., 2016, Effect of ramp-type heating on the vibration of functionally graded microbeams without energy dissipation, Mechanics of Advanced Materials and Structures, 23, 5, 529-537 